\title{
Development, Production, and Application of Recombinant Yeast Biocatalysts in Organic Synthesis
}

\author{
Roland Wohlgemuth*
}

\begin{abstract}
The use of biocatalysts from yeast strains in organic synthesis is well established and covers a broad range of reaction classes. A particularly interesting reaction class is the regio- and stereospecific attachment of sugar moieties (i.e. glycosylation) to a variety of natural products, from small molecules up to oligosaccharides and proteins. Since the bioactivity of many therapeutics depends on the proper glycosylation, the improvement of glycosylation methodology by chemical synthesis, biocatalysis or in vivo approaches is of major interest. We have developed glycosyltransferase-toolkits for the straight-forward and quantitative transfer of a specific monosaccharide moiety to an acceptor substrate. The stable expression of the $\beta(1 \rightarrow 4)$-galactosyltransferase I in Saccharomyces cerevisiae and $\alpha(1 \rightarrow 3)$-fucosyltransferase VI in Pichia pastoris has enabled these biocatalysts to be prepared in large-scale for use in organic synthesis. The application of galactosyltransferase using UDP-galactose and fucosyltransferase using GDP-fucose as NDP-sugar donor in the regio- and stereospecific galactosylation and fucosylation of small molecules is shown with a simplified reaction system. Optimisation of the reaction conditions allows for quantitative glycosylation reactions. The use of recombinant yeasts has been the key to performing this highly efficient glycosylation methodology and represents a building block of biocatalytic glycomics for the future.
\end{abstract}

Keywords: Fucosyltransferase · Galactosyltransferase - Quantitative glycosylation · Recombinant S. cerevisiae · Recombinant Pichia pastoris

Nomenclature

Ac acetyl

AOX alcohol oxidase

cDNA complementary DNA

CMP cytidin monophosphate

DNA deoxyribonucleic acid

Fuc fucose

FucT fucosetransferase

Gal galactosidase

GalT galactosyltransferase

GDP guanosine diphosphate

Glc glucose

$\mathrm{N} \quad n$ ucleoside

NDP nucleoside-diphosphate

$\mathrm{Neu} \quad$ neuramic acid

NTP nucleoside-triphosphate

PCR polymerase chain reaction

$\mathrm{Pi} \quad$ inorganic phosphate

UDP uridin diphosphate

\section{Introduction}

The development of yeast cultivations has resulted from the unique practical, cultural, and scientific innovations in the history of mankind starting from the need to prepare, maintain, and preserve food and drinks with pleasant flavour and nutritive characteristics [1][2]. Scientific innovation in biology and chemistry between the $17^{\text {th }}$ and the $19^{\text {th }}$ centuries and progress in instrumentation enabled knowledge to be transferred to technical and industrial applications using yeast as a chemical reagent at the beginning of the $20^{\text {th }}$ century, e.g. for the production of glycerol or ephedrine, as a reducing agent, as an oxidizing agent or to induce hydrolysis [2][3]. The use of whole yeast cells and yeast biocatalysts in organic synthesis has attracted considerable attention in respect of producing novel carbon-carbon bonds, secondary alcohols, and other optically active compounds used as chiral building blocks [4][5]. Because baker's yeast is inexpensive, readily available and the experimental application procedures for reductions are well-defined [6], this biocatalyst is popular among organic chemists [7-9]. Thus, it is not astonishing that it is the only biocatalyst listed in the encyclopaedia of reagents for organic synthesis [10]. The use of whole yeast cells or isolated enzymes in catalytic process technologies is also of continuing interest in order to minimize the amount of waste per kg of products [11]. Since classical yeast biocatalysts in general (Table 1) are non-toxic, easily biodegradable and not harmful to health, the biocatalytic process technology has the potential to fulfil all requirements for waste minimization, energy usage as well as reduction of safety, health, and environmental risks. With the availability of stable yeast biocatalysts, the synthe-

Table 1. Overview of classical yeast sources for biocatalysts in organic synthesis

\section{Species}

baker's yeast

brewer's yeast

Saccharomyces sp.

Candida sp.

Geotrichum sp.

Kluyveromyces sp.

Rhodotorula sp.

Yarrowia sp. 
sis of chiral compounds, metabolites, new chemical entities, and natural products by a combination of chemical and biological methods has become well established in the manufacturing process [12-14].

The number and types of reactions by yeast biocatalysts in organic synthesis is constantly increasing (a selection is shown in Table 2). Biocatalysts in general allow for an enormous diversity of chiral molecular recognition due to the chiral nature of the amino acid monomers (except glycine) and therefore represent privileged chiral catalysts. The applications in the synthesis of laboratory chemicals depend on a rapid and robust production technology for the required biocatalysts. It is therefore the vast knowledge of the chemistry, biochemistry, genetics, and genomics of yeast as well as the reaction engineering which make yeasts important organisms for the expression of biocatalysts for organic synthesis.

Table 2. Reactions catalyzed by classical yeast biocatalysts

Reactions catalyzed by yeast
carbonyl group reduction
activated double-bond reduction
oxidations
hydrolyses
asymmetric syntheses
cyclization reactions
asymmetric 1,3-dipolar cyclo-additions
Michael-type addition reactions
acyloin condensations

The field of glycosylation reactions is central to the synthesis of well-defined carbohydrates and glycoconjugates as well as to understanding their roles and structure-function relationships in a variety of biological areas such as infections, signal transduction, cell-cell interactions, host-pathogen interactions, inflammation, development, folding and stabilization, immune recognition, targeting proteins to their correct destination, tumour propagation and metastasis. The high information density, which is coded in the sequence and linkage of carbohydrates on the molecular scale, has led to an increased interest in the generation of new pharmacological agents [15]. Glycosylation reactions are therefore widely used in the synthesis of pharmaceuticals and bioactive compounds. Although the work on structure-function of carbohydrates goes back to the $19^{\text {th }}$ and $20^{\text {th }}$ centuries and a wealth of excellent methods have been developed, the greater structural complexity (i.e. due to branching and linkage diversity, chemo-, regio- and enantioselectivity) requires highly specific ana-



Fig. 1. Development of the number of publications appearing annually on the use of recombinant yeasts as biocatalysts

lytical and preparative tools. Considerable research efforts have recently been made to facilitate the analytical and synthetic processes [16][17]. In a purely chemical synthetic scheme, the use of protecting groups is inevitable and the overall sequence of steps for a specific glycosylation involves the preparation of glycosyl donors and glycosyl acceptors in a suitably protected form, the deprotection of the desired hydroxyl-group with the subsequent product separation after each step, the actual stereocontrolled glycosylation reaction followed by the final deprotection and finally product purification. Many advances have focussed on the simplification of this overall sequence by $e . g$. reducing the number of steps in the synthesis of donors and acceptors, minimizing protecting group manipulations, and simplifying the purification steps [17]. The automation of the synthetic steps as in peptide and nucleic acid synthesis is very promising [18]. The formation of a glycosidic bond between a glycosyl donor and a glycosyl acceptor with enzymes such as glycosidases [19][20] and glycosyltransferases [21-24] offers simple and highly effective synthetic tools for a variety of practical syntheses. Furthermore, they have the potential to fulfil the waste minimization criteria mentioned above. Enzymatic synthesis using glycosyltransferases have the advantages that no chemical protection and deprotection is required, that an increasing number of glycosyl donors used as building blocks is easily available [25] and that the reaction proceeds as in vivo with absolute regio- and stereospecificity under mild reaction conditions [16]. This is an advantage if the acceptor is susceptible to modifica- tions as are many natural products [26], peptides and proteins [27] and it further reduces the challenges in the purification of the products.

\section{Development of Recombinant Yeast Biocatalysts}

Although a variety of natural yeast strains have been in routine use as biocatalysts for organic reactions, the availability of efficient genetic tools for overexpression and gene disruption has added another dimension for the development of yeast biocatalysts (e.g. shown in Fig. 1 by the increase in the number of papers on recombinant yeast biocatalysts). As more yeast genomes are published, comprehensive databases provide sequences, functional annotations and interactions [28][29]. Coupled with expression and protein abundance data in Saccharomyces cerevisiae, protein evolution occurs at rates largely unrelated to their functions and a slow evolution of highly expressed proteins has been suggested [30]. The combination of genome database information with biotransformation data on a molecular level is not only useful in the understanding and engineering of metabolic pathways, but also for the development of yeast biocatalysts.

These tools together with the long and extensive experience in yeast cultivation make yeast expression systems very attractive for the expression of a large variety of biocatalysts. The expression of selected biocatalyst genes in a suitable host such as Saccharomyces cerevisiae [31][32], Pichia pastoris [33-36], Hansenula polymorpha 
[37] [38] or non-conventional yeasts [39] is very attractive for mammalian enzymes.

The large-scale production of biocatalysts is facilitated by the construction of genetically stable recombinant strains overexpressing active enzyme in a reproducible way and by yielding a high final activity per volume which can be carried through downstream processing. This is especially important for proteins which are present in nature only in small amounts and for proteins with low expression, different glycosylation patterns or folding bottlenecks in other expression systems. The universe of natural glycosyltransferases is of considerable fundamental interest [40]. However, the isolation from natural sources involves large amounts of raw material and lengthy purification sequences. It is therefore essential to develop generalized gene expression technology to prepare glycosyltransferases in substantial amounts in order to resolve the availability problem of these unique enzymes. Yeast cell hosts offer good opportunities for the expression of these glycosyltransferases [41] because higher eukaryotic cell hosts may express silenced genes and yeasts have only a restricted number of their own glycosyltransferases [42]. A variety of plasmid DNA vectors which are capable of transforming auxotrophic yeast strains and which have also bacterial sequences that can be selected for and propagated in E.coli, are suitable for subcloning the glycosyltransferase cDNA and represent important screening tools [43]. The identification of positive transformants of yeast host strains like Pichia pastoris KM71 (arg4his4aox1: ARG4) by direct PCR screening and the inclusion of yeast promoter and terminator sequences for efficient transcription have been used in the construction of recombinant Pichia pastoris strains [44]. It is useful for downstream processing of soluble forms of glycosyltransferases that the vector contains a signal sequence such as the N-terminal signal sequence of Saccharomyces cerevisiae $\alpha$-factor for the secretion of the expressed protein into the medium. This is therefore the preferred method to prepare enough of such isolated biocatalysts to study their functions or to apply for analytical or preparative purposes although biocatalysts can also be isolated if yeast cells have to be broken. Another strategy aims at expressing glycosyltransferases immobilized to the yeast cell wall [45][46]. In the case of glycosyltransferases, the yeast expression systems have been important for developing a series of soluble functional biocatalysts [44][47]. For the human $\beta(1 \rightarrow 4)$-galactosyltransferase gene, the Saccharomyces cerevisiae expression system has been developed. The growth of this yeast was controlled with a fedbatch procedure, which demonstrated for the first time that heterologous expression of a gly- cosyltransferase is possible on a large scale [48]. The expression of the human $\alpha(1 \rightarrow$ 3)-fucosyltransferase VI gene has been developed in the Pichia pastoris system with the alcohol-oxidase (AOX) promoter in the protease-negative KM71-host in shake flasks [44]. The development of efficient fermentation and downstream processing required the development of sensitive and reliable non-radioactive activity assays for in-process control. These analytical tools together with the on-line monitoring of fermentation parameters have been the prerequisites for the development of a successful large-scale production procedure for these glycosyltransferases. In the case of the Saccharomyces cerevisiae expression system, additional monitoring and control of residual glucose is essential. In the case of the Pichia pastoris expression system a sensor using well-established semi-conductor technology, which after calibration at the required temperature (Fig. 2) allows a reproducible control of the residual methanol concentration during heterologous protein expression, has become standard [49]. Pichia pastoris not only grows on methanol, but methanol also triggers the AOXpromoter and high concentrations of it can compromise the cells. The expression as a function of the methanol concentration, $\mathrm{pH}$, temperature and media components can then be optimized and enables the development of a robust cultivation procedure. The downstream processing steps depend on the application of the glycosyltransferases. Generally speaking, glycosidases and proteases have to be removed in order to be useful for high-yield synthesis and obtain the maximum yield of product per unit of

\section{Production of Recombinant Yeast Biocatalysts}

In large-scale we have produced a series of glycosyltransferases with different bond specificities, such as $\beta(1 \rightarrow 4)$-galactosyl-transferase I and $\alpha(1 \rightarrow 3)$-fucosyl-transferase VI, in stable and usable form for carbohydrate synthesis and the glycosylation of natural products. Thereby the emphasis has been on reproducible expression and robust processes. The main factors influencing successful and reproducible expression of glycosyltransferases in yeast include (i) establishing rapid glycosyltransferase activity assays enabling expression and downstream processing to be monitored, (ii) stable constructs and proper expression conditions which keep the activities stable over the required time of expression, (iii) adaptation of the process control technology, (iv) stability testing and establishing the proper downstream processing. Furthermore, the use of additional on-line control equipment (e.g. for the measurement and control of glucose and methanol) is absolutely essential for consistent and scalable operations in production. As the causes of proteolytic degradation of secreted recombinant proteins in Pichia pastoris have been attributed to methanol metabolism along with cell lysis towards the end of fermentation [50], generalized approaches to maximize yields from fedbatch processes [51]

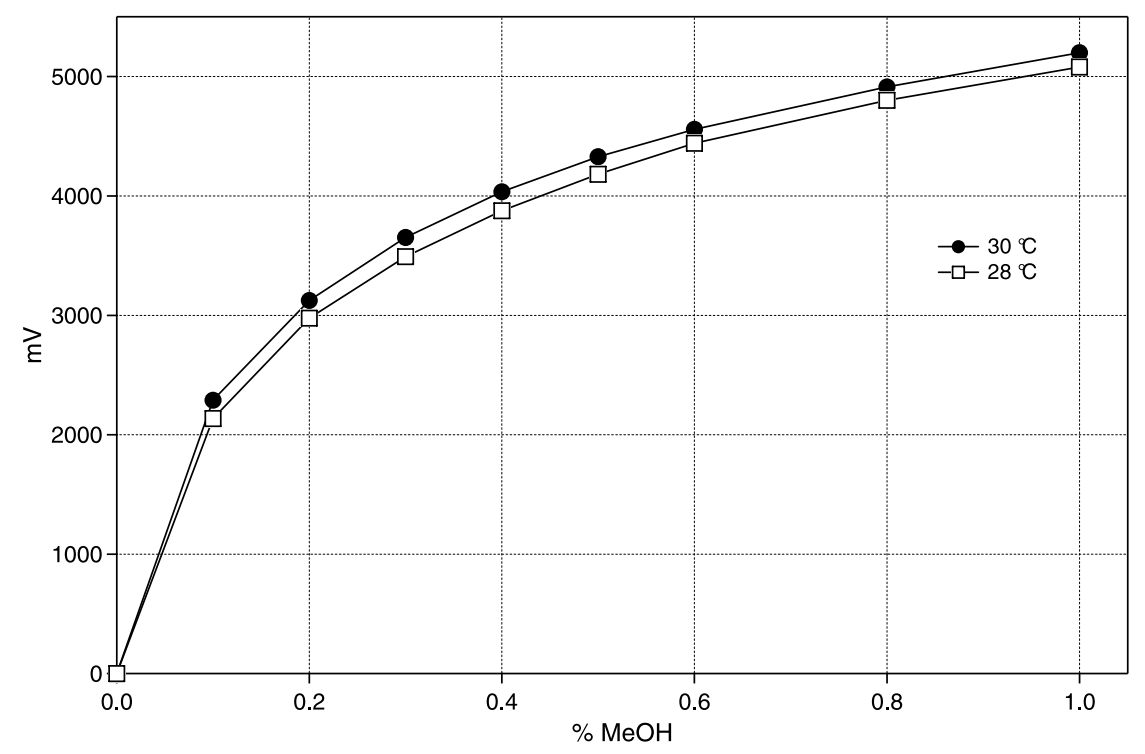

Fig. 2. Calibration-curve of on-line methanol (Raven Biotech Inc., Vancouver, Canada) sensor used in feedback control loop 
together with in-process controls of expression and precautionary measures such as the standard use of protease inhibitors during downstream processing move these productions towards efficient, reproducible large-scale operations.

\section{Applications of Recombinant Yeast Biocatalysts}

The scope of preparative applications is expanding rapidly along many different reaction classes. In carbohydrate synthesis and glycosylation of natural products [52][53], a series of glycosyl donors (Table 3 ), with different reaction conditions and different application strategies for glycosyltransferases have been used (see Scheme 1 for an example of a $\beta(1 \rightarrow 4)$-galactosylation of N-acetyl-D-glucosamin). Since $\beta(1 \rightarrow 4)$-galactosyltransferase is available from bovine milk, it has become one of the best-studied glycosyltransferases and Scheme 2 shows the wide range of variation that is tolerated by the enzyme on the acceptor side. In order to simplify the applications for the research scale, we supplied the glycosyl donor directly instead of in situ preparation with another enzyme system and omitted the regeneration of the nucleoside diphosphate. The first glycosylation kits worldwide have been assembled by reducing system complexity and removing the inhibiting nucleotide diphosphate with alkaline phosphatase as seen by the $\beta(1 \rightarrow$ 4)-galactosylation in Scheme 3 (case A). The first three kits involve $\beta(1 \rightarrow 4)$-galactosylation, $\alpha(1 \rightarrow 3)$-galactosylation and $\alpha(1 \rightarrow$ $3)$-fucosylation. The large-scale availability of $\alpha(1 \rightarrow 3)$-fucosyltransferase now enables the substrate investigations on the structural requirements of the acceptor to be extended (Scheme 4). The reactions can be quickly run to completion and with absolute specificity, therefore eliminating the need to purify closely related products. The threestep sequence of protection-glycosidic bond formation-deprotection has thus been

Table 3. Overview of nucleotide sugars as glycosyl-transfer reagents

Nucleotide sugar
UDP-galactose
UDP-glucose
UDP-glucuronic acid
UDP-N-acetyl-galactosamin
UDP-N-acetyl-glucosamin
UDP-xylose
GDP-fucose
GDP-mannose
CMP-N-acetyl-neuraminic acid

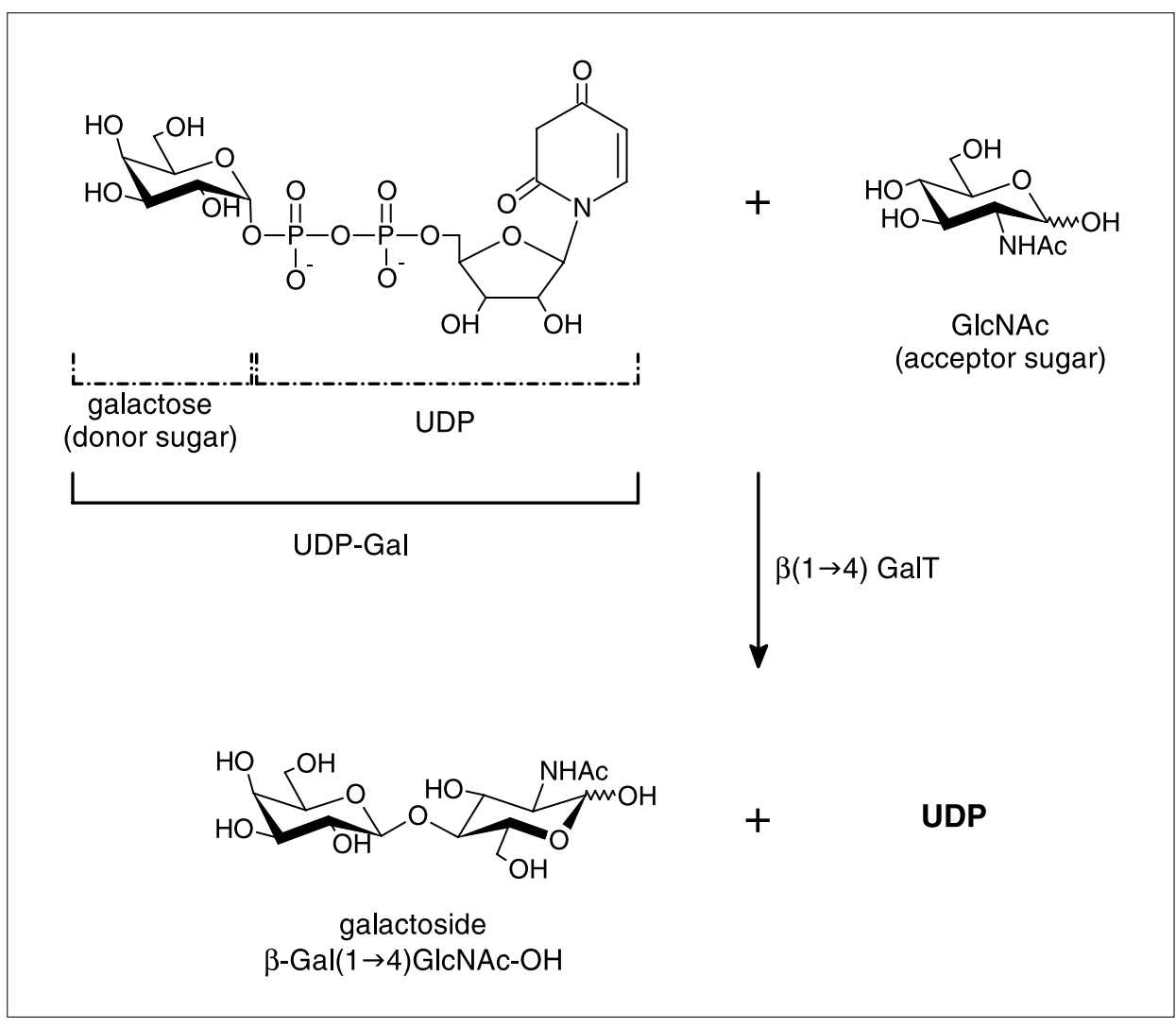

Scheme 1. $\beta(1 \rightarrow 4)$-Galactosylation of N-acetyl-D-glucosamin (GlcNAc)

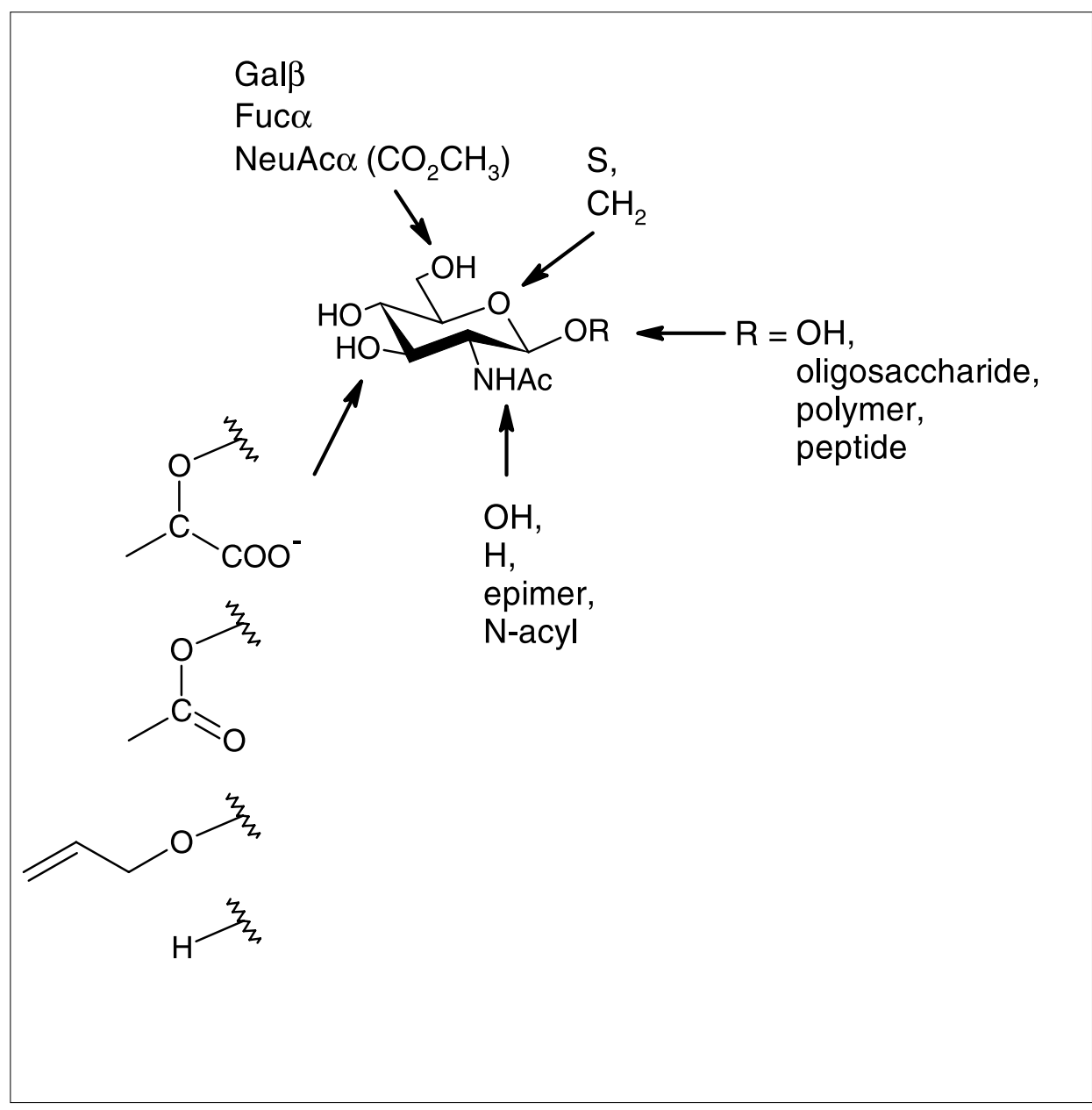

Scheme 2. Modifications of GlcNAc as acceptors in $\beta(1 \rightarrow 4)$ GalT-catalyzed transfers of galactose 
(A)

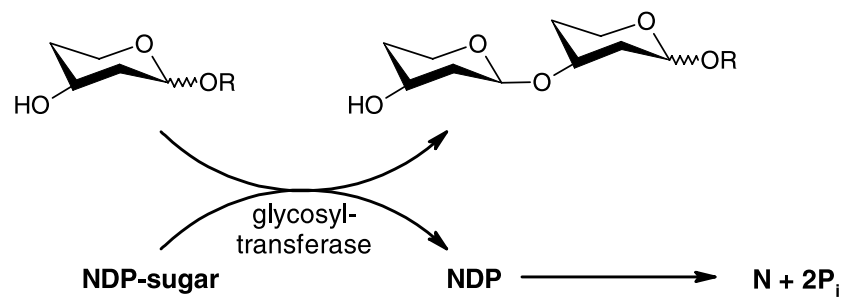

(B)

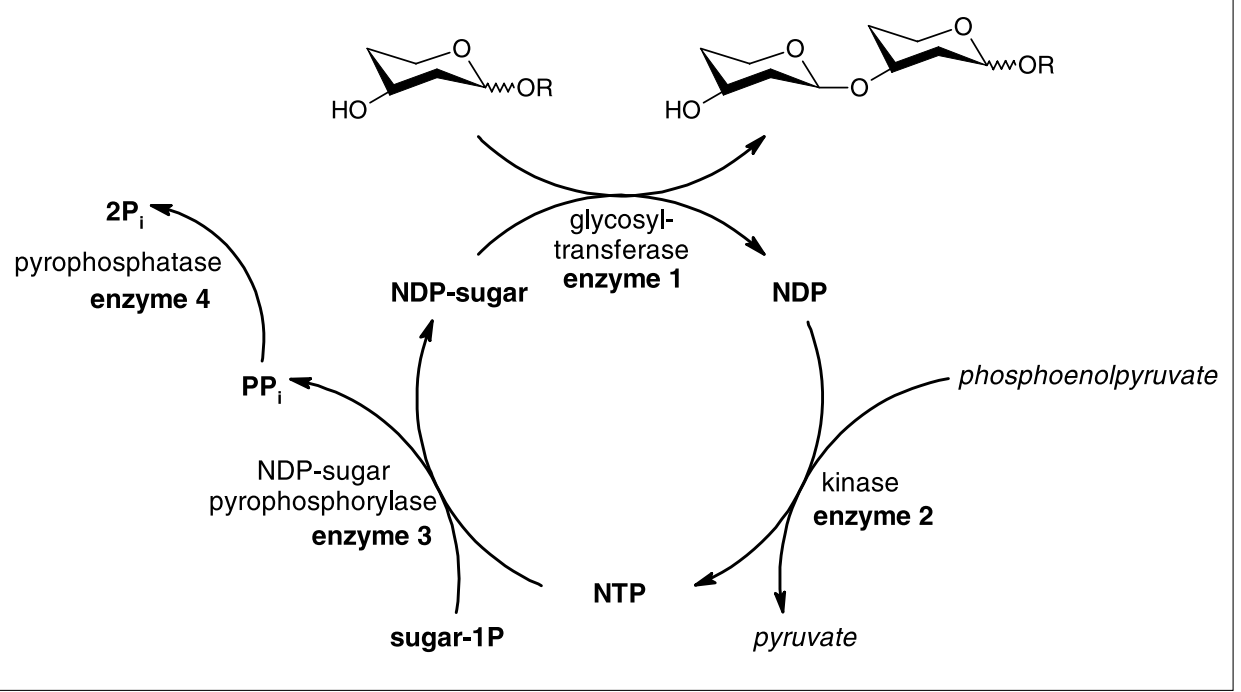

Scheme 3. Methods for avoiding product inhibition in glycosyltransferase-catalyzed synthesis (A) addition of phosphatase $(B)$ recycling of sugar nucleotides

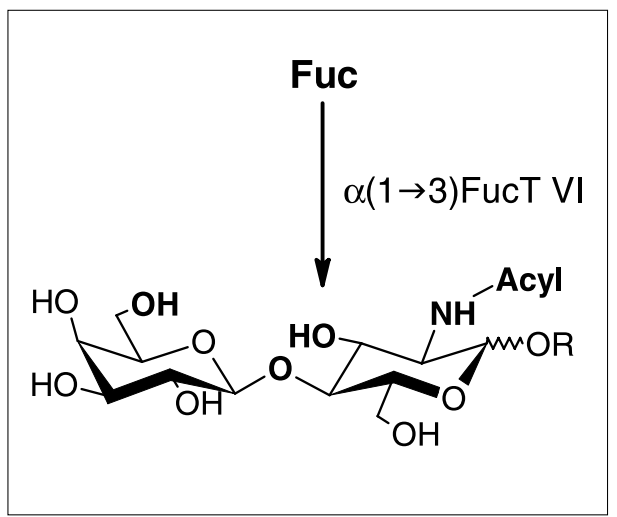

Scheme 4. Modifications of $\mathrm{N}$-acyllacetosamin as acceptors in $\alpha(1 \rightarrow 3)$ FucT Vl-catalysed transfers of fucose (Minimum structural requirements for an acceptor are: 6'-OH-group, 3-OH-group, $\beta$ 1,4-linkage, 2-NH-acylation)

condensed into one enzymatic step and the tools for these specific biocatalytic glycosylations can be used worldwide [54].

\section{Discussion}

Future success in the domain of yeast biocatalysts in organic synthesis requires an integrated approach from biology, chemistry, and engineering, taking advantage of the progress in each of these disciplines. The opportunities for new yeast biocatalytic processes are remarkable and have gained new support due to the results of yeast genome projects, the discovery of novel biocatalyst functions and improvements in yeast expression systems.

In the field of biocatalytic glycosylation we are at the beginning of a development for which the cornerstones have been laid. Beside glycosyltransferases from mammalian and bacterial sources, the expression of human glycosyltransferases is of much interest.

A modular system approach consisting of matching pairs of new recombinant glycosyltransferases and the required nucleotide sugars has tremendous potential. Saccharomyces cerevisiae and Pichia pastoris are versatile host systems for recombinant glycosyltransferase production. Furthermore, the expression of new glycosyltransferases is facilitated by reliable high-throughput screening.

The improved synthetic methods using biocatalytic and automated chemical synthesis and the concomitant miniaturization of analyses in microtiter plates [55] and microarrays [56] offer exciting opportunities to decipher the glycocode and use it as a tool in the rapidly growing discipline of glycomics [57]. The use of yeast biocatalysts will continue to grow to provide convenient solutions to certain expression problems in viral systems and insect cells, to change glycosylation patterns, to allow the number of synthetic tools used in organic synthesis to be increased and, finally, to make use of nature's biocatalysts to create the molecules needed to solve our global problems.

\section{Acknowledgements}

I would like to thank Reto Schüpbach, Peter Jani, Andreas Naundorf, Karin Koller, Daniel Jaquemar, Wolfgang Hass, and Klaus Trummler at Fluka for their dedicated work as well as Eric Berger, Martine Malissard, and Maud Ramuz at the University of Zurich for their excellent cooperation, and the Swiss National Science Foundation's SPP Biotechnology programme for supporting project no. 5002-57797. In addition, I would like to thank Robert Lovchik and Karin Kovar for their help in the publication process.

Received: August 5, 2005

[1] A.H. Rose, J.S. Harrison, 'The Yeasts', Vol. I-III, Second edition, Academic Press, New York, 1987.

[2] J.F.T. Spencer, D.M. Spencer, 'Yeasts in Natural and Artificial Habitats', SpringerVerlag, Berlin, 1997.

[3] A.H. Cook, 'The Chemistry and Biology of Yeasts', Academic Press, New York, 1958.

[4] J.A. Barnett, R.W. Payne, D. Yarrow, 'Yeasts characteristics and identification', Cambridge University Press, Second edition, 1990.

[5] V. Prelog, Pure and Applied Chemistry 1964, 9, 119.

[6] D. Seebach, M.A. Sutter, R.H. Weber, M.F. Züger, Org. Synth. 1985, 63, 1.

[7] S. Servi, Synthesis 1990, 1.

[8] T. Sugai, Curr. Org. Chem. 1999, 3, 373.

[9] K. Nakamura, R. Yamanaka, T. Matsuda, T. Harada, Tetrahedron: Asymmetry 2003, $14,2659$.

[10] L.A. Paquette (Ed.), 'Encyclopedia of Reagents for Organic Synthesis', 1995, Vol.1, 233.

[11] R.A. Sheldon, Pure Appl. Chem. 2000, 72(7), 1233.

[12] K. Drauz, H. Waldmann, 'Enzyme Catalysis in Organic Synthesis: A Comprehensive Handbook', Second edition, Vol. I-III, Wiley-VCH, Weinheim, 2002.

[13] A. Liese, K. Seelbach, C. Wandrey, 'Industrial Biotransformations', Wiley-VCH, Weinheim, 2000.

[14] H.U. Blaser, E. Schmidt, 'Asymmetric Catalysis on Industrial Scale', Wiley-VCH, Weinheim, 2004.

[15] C.H. Wong (Ed.), 'Carbohydrate-Based Drug Discovery', Wiley-VCH Verlag, Weinheim, Vol. 1-2, 2003. 
[16] B. Ernst, G.W. Hart, P. Sinaÿ (Eds.), 'Carbohydrates in Chemistry and Biology', Wiley-VCH, Weinheim, Part I and II, Vol. $1-4,2000$.

[17] B. Fraser-Reid, K. Tatsuta, J. Thiem (Eds.), 'Glycoscience: Chemistry and Chemical Biology', Springer-Verlag, Berlin, Vol. I-III, 2001.

[18] P.H. Seeberger, Chem. Comm. 2003, 1115.

[19] G. Perugino, B. Cobucci-Ponzano, M. Rossi, M. Moracci, Adv. Synth. Catal. 2005, 347, 941.

[20] V. Kren, J. Thiem, Chem. Soc. Rev. 1997, 26, 463.

[21] K.M. Koeller, C.H. Wong, Chem. Rev. 2000, 100, 4465.

[22] S. Hanson, M. Best, M.C. Bryan, C.H. Wong, Trends Biochem. Sci. 2004, 29, 656.

[23] Z. Zhang, J. Gildersleeve, Y.Y. Yang, R. Xu, J.A. Loo, S. Uryu, C.H. Wong, P.G. Schultz, Science 2004, 303, 371.

[24] M.M. Palcic, O. Hindsgaul, Trends Glycosci. Glycotech. 1996, 8(39), 37.

[25] S. Koizumi, T. Endo, K. Tabata, A. Ozaki, Nat. Biotechnol. 1998, 16, 847.

[26] J.S. Thorson, J. Thomas, J. Jiang, J.B. Biggins, J. Ahlert, Curr. Org. Chem. 2001, 5 , 139.

[27] C.H. Wong, J. Org. Chem. 2005, 70, 4219.

[28] U. Gueldener, M. Muensterkoetter, G. Kastenmueller, N. Strack, J. van Helden, C. Lerner, J. Richelles, S.J. Wodak, J. Garcia-Martine, J.E. Perez-Ortin, H. Miachel, A. Kaps, E. Talla, B. Dujon, B. Andre, J.L. Souciet, J. De Montigny, E. Bon, C. Gallardin, H.W. Mewes, Nucl. Acids Res. 2005, 33, D364.

[29] http://mips.gsf.de/genre/proj/yeast and http://genome-www.stanford.edu/saccharomyces/
[30] D.A. Drummond, J.D. Bloom, C. Adami, C.O. Wilke, F.H. Arnold, Quant. Biol. 2005, 1.

[31] B. Prinz, J. Schultchen, R. Rydzewski, C. Holz, M. Boettner, U. Stahl, C. Lang, J. Struct. Funct. Genomics 2004, 5, 29.

[32] S. Macauley-Patrick, M.L. Fazenda, B. McNeil, L.M. Harvey, Yeast 2005.

[33] G.P. Lin Cereghino, J. Lin Cereghino, C. Ilgen, J.M. Cregg, Curr. Opin. Biotechnol. 2002, 13, 329.

[34] J.M. Cregg, J. Lin Cereghino, J. Shi, D.R Higgins, Mol. Biotechnol. 2000, 16, 23.

[35] List of heterologous proteins expressed in Pichia pastoris: http://faculty.kgi.edu/ cregg

[36] M. van der Heide, M. Veenhuis, I. van der Klei, Topics Cur. Gen. 2003, 2, 207.

[37] G. Gellissen, L. Scheffers, FEMS Yeast Res. 2003, 4, 129.

[38] G. Gelissen (Ed.), 'Hansenula polymorpha: Biology and Applications', WileyVCH Verlag, Weinheim, 2002.

[39] K. Wolf, K. Breunig, G. Barth, 'Non-Conventional Yeasts in Genetics, Biochemistry and Biotechnology', Springer-Verlag, Berlin, 2003.

[40] N. Taniguchi, K. Honke, M. Fukuda (Eds.) 'Handbook of Glycosyltransferases and Related Genes', Springer-Verlag, Tokyo, 2002.

[41] M. Bencúrová, D. Rendic, G. Fabini, E.M Kopecky, F. Altmann, I.B.H. Wilson, Biochimie 2003, 85, 413

[42] M.A. Kukuruzinska, M.L.E. Bergh, B.J. Jackson, Ann. Rev. Biochem. 1987, 56, 915.

[43] J.H. Nett, N. Hodel, S. Rausch, S. Wildt, Yeast 2005, 22, 295.

[44] M. Malissard, S. Zeng, E.G. Berger, Biochem. Biophys. Res. Comm. 2000, 267, 169.
[45] H. Abe, Y. Shimmo, Y. Jigami, Glycobiol. 2003, 13, 87.

[46] H. Salo, E. Sievi, T. Suntio, M. Mecklin, P. Mattila, R. Renkonen, M. Makarow, FEMS Yeast Res. 2005, 5, 341.

[47] M. Malissard, S. Zeng, E.G. Berger, Glycoconj. J. 1999, 16, 125.

[48] G.F. Herrmann, C.H. Krezdorn, M. Malissard, R. Kleene, H. Paschold, D. Weuster-Botz, U. Kragl, E.G. Berger, C. Wandrey, Prot. Express. Purif. 1995, 6 , 72.

[49] B.E. Mayson, D.G. Kilburn, B.L. Zamost, C.K. Raymond, G.L. Lesnick, Biotechnol. Bioeng. 2003, 81, 291.

[50] J. Sinha, B.A. Plantz, M. Inan, M.M. Meagher, Biotechnol. Bioeng. 2004, 89, 102.

[51] W. Zhang, J. Sinha, L.A. Smith, M. Inan, M.M. Meagher, Biotechnol. Prog. 2005, 21, 386.

[52] Z. Guo, P.G. Wang, Appl. Biochem. Biotechnol. 1997, 68, 1.

[53] S. Riva, Curr. Opin. Chem. Biol. 2001, 5, 106.

[54] Instructions for use of the glycosylation kits: http://www.sigma-aldrich.com

[55] M.C. Bryan, F. Fazio, H.K. Lee, C.Y. Huang, A. Chang, M.D. Best, D.A. Calarese, O. Blixt, J.C. Paulsen, D. Burton, I.A. Wilson, C.H. Wong, J. Am. Chem. Soc. 2004 , 126,8640 .

[56] O. Blixt, S. Head, T. Mondala, C. Scanlan, M.E. Huflejt, R.Alvarez, M.C. Bryan, F. Fazio, D. Calarese, J. Stevens, N. Razi, D.J. Stephens, J.J. Skehel, I. van Die, D.R. Burton, I.A. Wilson, R. Cummings, N. Bovin, C.H. Wong, J.C. Paulsen, Proc. Nat. Acad. Sci. USA 2004, 101, 17033.

[57] D.M. Ratner, E.W. Adams, M.D. Disney, P.H. Seeberger, Chem. Biochem. 2004, 5 , 1375. 